\title{
Unmoderated Posters: Miscellaneous
}

\section{UP-64}

Should We as Surgeons Worry about Perceived Coercion by the in Clinical Decision to Operate in Non-absolute Indication for Surgery?

Anwar, Adeel; Ahmed, Munir; Khakwani, Alex

Princess Royal University Hospital, Orpington, Kent, United Kingdom Introduction and Objectives: Shared decision making is the key of modern day medical practice. We believe in patients' empowerment and their right to choose or refuse the offer treatment. It is interesting to know despite of signing consent form for a surgical procedure number of patients remains unclear and consider it against their free will. This is also relevant to urological procedures where the indications are not absolute, either because of availability of alternative treatments, elderly patients and quality of life issues which may affect treatment choices. As per United Kingdom Mental Capacity Act (MCA 2005) 3 elements of the decision making capacity need to be fulfilled: (i) comprehension/understanding of information; (ii) retaining information; (iii) using or weighing up information in decision making process.

Methods: This study was conducted on 50 patients attending urology preassessment clinic who were scheduled for LUTS surgery with non-absolute indications (patients with a catheter in-situ, or a past medical history of prostate surgery/cancer were excluded). Cognition and capacity of these patients were assessed by 30 points mini mental state score (MMSE). A validated Perceived Coercion Questionnaire assessed coercion in the decision making process against variables of freedom, choice, idea, control and influence in the decision to have treatment.

Results: This was a prospective study including 50 male patients with LUTS mean age of 67.3 year.42 patients underwent transurethral resection of prostate (TURP), 4 had bladder neck incision and 4 had Green light laser prostatectomy. Cognition-mean MMSE score $=28.2$ (no patient scored below 22). The mean perceived coercion score was 0.42 (maximum possible of 5 ). Conclusions: All these patients had normal cognition and were therefore deemed to possess capacity to consent to the specified procedure. Furthermore the study showed that there was no evidence to suggest coercion in the decision making process.

\section{UP-65}

\section{Prostate Cancer Awareness Campaign and the Volume of Public} Internet Searches for Disease and Screening Information

Veldhoen, Richard; ${ }^{1}$ Matta, Rano ${ }^{2}$; Leveridge, Michael ${ }^{1}$

${ }^{1}$ Queen's University, Kingston, ON, Canada; ${ }^{2}$ University of Toronto, Toronto, ON, Canada

Introduction and Objectives: Patient advocacy and research agencies commonly employ public campaigns such as "awareness months" to fundraise or to promote health or behaviour change. Quantifying the public response may be challenging. We analyzed Canadian internet search volume data on prostate cancer \& screening over time, related to known awareness campaigns, as a marker of their impact
Methods: The web service Google Trends was used to identify relative search volumes for the term "prostate cancer" on a monthly basis over the past 10 years. We searched subsequently for "PSA test" and "prostate specific antigen". For comparison we also analyzed searches for breast cancer, mammography, colon cancer and colonoscopy. Volumes were analyzed by month based on the relative volume for that year

Results: Searches for prostate cancer were significantly higher in November than in any other month, particularly in the past 5 years $(19.8 \%$ above the yearly mean; 2004-2008: $2.41 \%$ >mean, 2009-2013: $37.3 \%$ >mean), corresponding to the "Movember" campaign (Fig. 1). There was no increase in search volume during September, which is Prostate Cancer Awareness Month. Searches for PSA testing were not increased in November. By contrast, searches for breast cancer rose sharply in October (Breast Cancer Awareness Month) vs. other months ( $41.3 \%$ above year mean); searches for "mammogram" were also highest in October (16.8\% above mean). Breast cancer search volume was always higher than prostate cancer search volume (mean 289\% higher; Fig. 2). There was no increased search volume for colon cancer or colonoscopy in March (Colon Cancer Awareness Month). Conclusions: There is a significant increase in Internet searches for prostate cancer in November, which corresponds to the popular awareness campaign Movember. This increase in search volume does not extend to increased searches for PSA testing. This may inform tailoring of messaging during these campaigns.

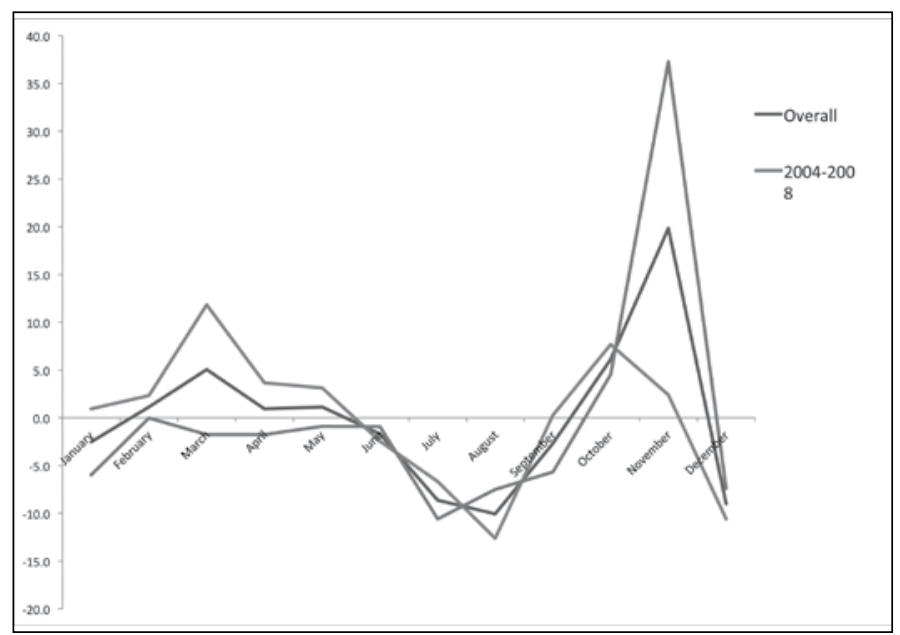

Fig. 1. UP-65. Mean monthly Google search volumes for the term "prostate cancer," as a percentage of an individual year's mean search volume. 